Supporting Information

\title{
Quantitative cooperative binding model for intrinsically disordered proteins interacting with nanomaterials
}

\author{
Da-Wei Li, ${ }^{1 *}$ Mouzhe Xie, ${ }^{2}$ and Rafael Brüschweiler ${ }^{1,2,3^{*}}$ \\ ${ }^{1}$ Campus Chemical Instrument Center, The Ohio State University, Columbus, Ohio \\ 43210, USA \\ ${ }^{2}$ Department of Chemistry and Biochemistry, The Ohio State University, Columbus, \\ Ohio 43210, USA \\ ${ }^{3}$ Department of Biological Chemistry and Pharmacology, The Ohio State University, \\ Columbus, Ohio 43210, USA \\ ${ }^{*}$ To whom correspondence should be addressed: \\ Da-Wei Li, Ph.D., E-mail: lidawei@gmail.com \\ Rafael Brüschweiler, Ph.D., E-mail: bruschweiler.1@osu.edu
}




\section{MATERIALS AND EXPERIMENTAL METHODS}

Sample preparation. The expression protocol of ${ }^{15} \mathrm{~N}$ or ${ }^{15} \mathrm{~N},{ }^{13} \mathrm{C}$ isotopically-labeled IDPs, including the transactivation domain of human tumor suppressor p53 (p53TAD), human $\alpha$ synuclein (aSyn), prokaryotic ubiquitin-like protein (Pup), and the cytoplasmic segment 2 at the C-terminus of the canine $\mathrm{Na}^{+} / \mathrm{Ca}^{2+}$ exchanger (Cyto2), was reported previously. ${ }^{[1,2]}$ Site-directed mutagenesis was conducted following QuikChange Lightning protocol (Agilent Technologies). After purification using Ni-NTA agarose (QIAGEN GmbH) affinity columns, the His ${ }_{6}$-tag of IDP constructs were cleaved by tobacco etch virus (TEV) protease. The extinction coefficients (in unit of $\mathrm{M}^{-1} \mathrm{~cm}^{-1}$ ) at $\mathrm{A}_{280}$ are 11380 for p53TAD and its mutants, 5120 for aSyn and its mutants, 1280 for Pup, and 5690 for Cyto2, which were used to estimate protein concentration. The final samples all consist of $170 \mu \mathrm{M}$ IDPs in $20 \mathrm{mM}$ sodium phosphate buffer (pH 7.0, also contains $100 \mathrm{mM} \mathrm{NaCl}$ and 10\% $\mathrm{D}_{2} \mathrm{O}$ ). For SNP-doped samples, as-dialyzed colloidal SNPs (AkzoNobel Bindzil 2040, $19.5 \pm 5.3 \mathrm{~nm}$ in diameter by transmission electron microscopy and $25.3 \pm 8.9 \mathrm{~nm}$ by dynamic light scattering, $\zeta$-potential $-23.0 \pm 7.4 \mathrm{mV}$ at $\mathrm{pH} 7.0^{[3]}$ ) were directly mixed with IDP solutions at desired concentrations that are specified in the main text. Equilibria were established immediately, and all samples were stable over the entire course of NMR measurements.

NMR relaxation experiments. Backbone amide ${ }^{15} \mathrm{~N}-\mathrm{NMR}$ spin relaxation of each IDP was measured by ${ }^{15} \mathrm{~N}-{ }^{1} \mathrm{H}$ HSQC-based pseudo-3D experiments, where eight (or six for some samples) relaxation delay intervals were employed. ${ }^{[2]}$ The residue-specific relaxation parameters, $R_{1}$ and $R_{1} \rho$, were extracted by fitting the intensity $v s$. delay data with a single exponential, and the transverse relaxation rates, $R_{2}$, were subsequently calculated based on the ${ }^{15} \mathrm{~N}$ spin-lock field strength $(2047 \mathrm{~Hz})$ and resonance offset of each cross-peak. The final errors associated with these rates that were estimated based both on the cross-peak intensity fitting and the relaxation exponent fitting are typically less than $10 \%$. The difference of $R_{2}$ rates between SNP-present and SNP-absent samples were denoted $\Delta R_{2}$ and were plotted against IDP primary sequence, known as an "interaction profile". All NMR experiments were conducted on a Bruker Avance III HD spectrometer (equipped with a TCI cryogenic probe) operated at $850 \mathrm{MHz}{ }^{1} \mathrm{H}$ frequency at 298 $\mathrm{K}$. These relaxation data are highly reproducible as demonstrated in Figure S2. 
Table S1. Sequences of wild-type IDPs used in current study ${ }^{a}$

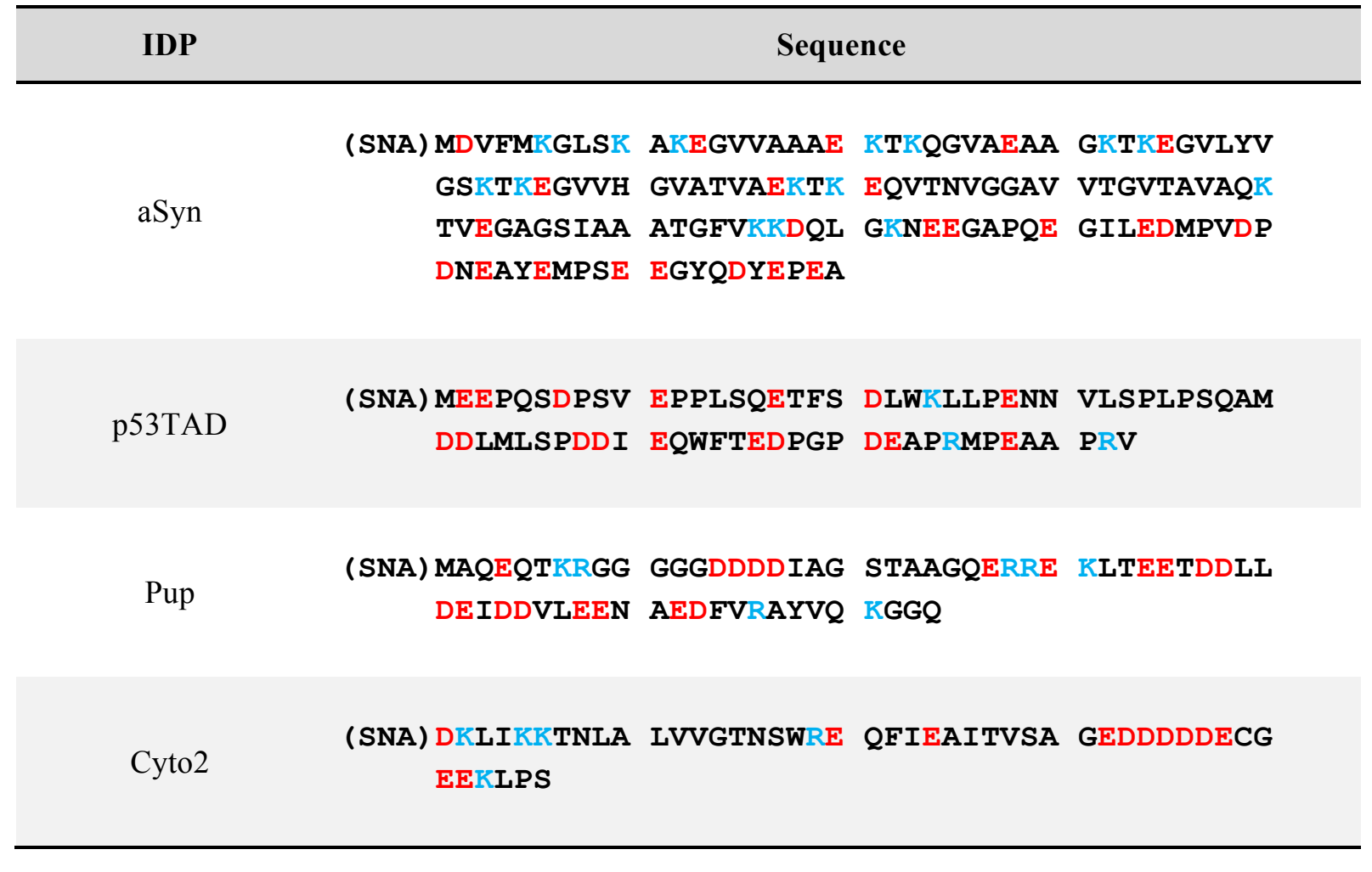

${ }^{a}$ Positively charged residues ( $\mathrm{R}$ and $\mathrm{K}$ ) are color-coded in blue and negatively charged residues (D and $\mathrm{E}$ ) are in red. The N-terminus of each IDP contains three non-native residues "SNA" after TEV-cleavage, and their contributions to the surface binding was taken into account in calculations using the SILC model. 
Table S2. SNP concentrations in IDP samples.

\begin{tabular}{ccc}
\hline IDP & Construct & [SNP] $(\mu$ M) \\
\hline \multirow{2}{*}{ p53TAD } & WT & $0.84,1.68$, and 2.52 \\
& K24N & 2.52 \\
& P27N & 2.52 \\
& D48N/D49N & 2.52 \\
aSyn & WT & 0.084 and 0.34 \\
& K21N & 0.17 \\
Kup & K21N/K45N & 0.17 \\
Cyto2 & WT & 0.17 \\
\hline
\end{tabular}



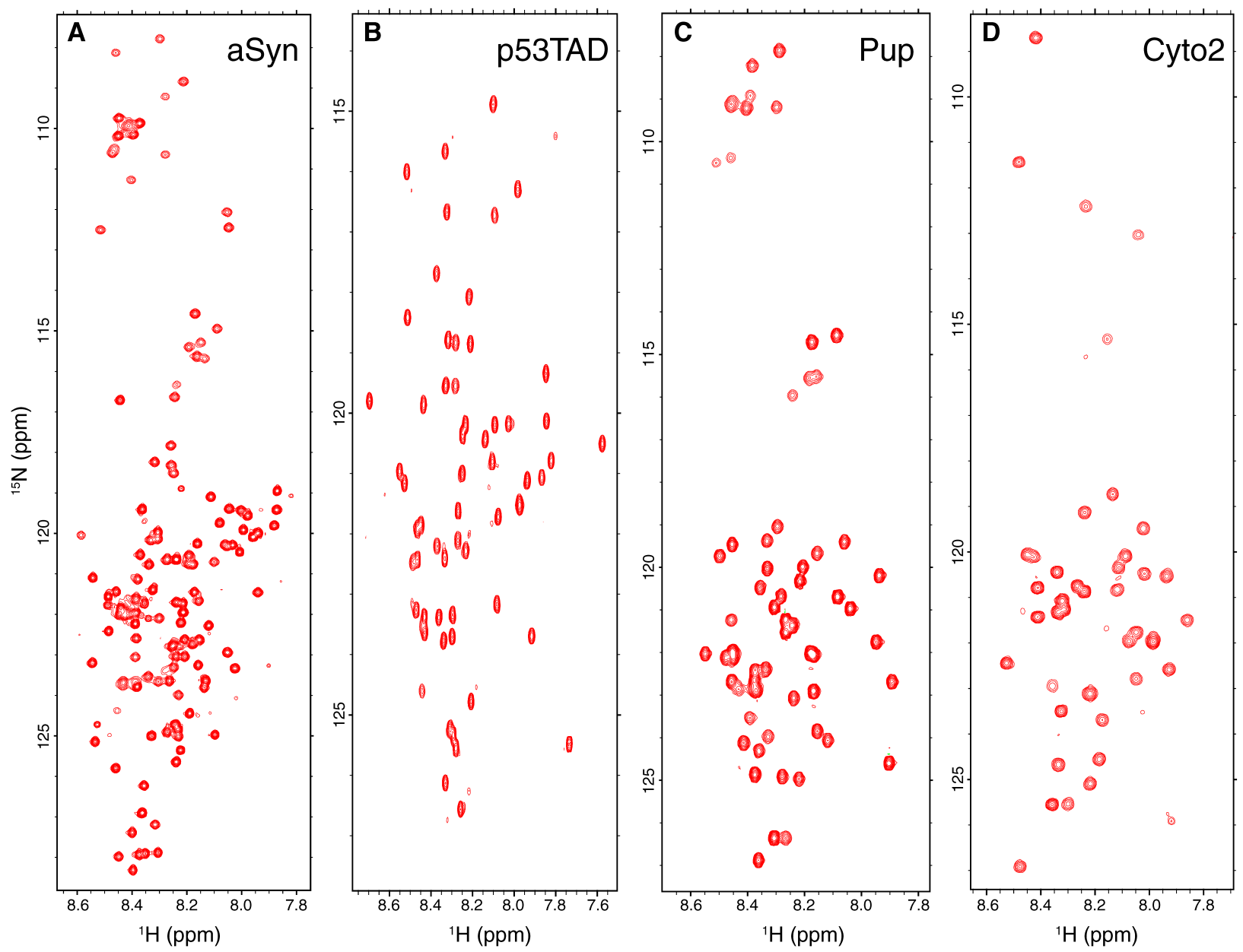

Figure S1. Backbone regions of representative ${ }^{15} \mathrm{~N}-{ }^{1} \mathrm{H}$ HSQC spectra of (A) aSyn, (B) p53TAD, (C) Pup, and (D) Cyto2. NMR relaxation experiments based on these ${ }^{15} \mathrm{~N}-{ }^{1} \mathrm{H}$ HSQC spectra hence provide siteresolved (i.e. residue-specific) information that enables quantitative measurements of IDP-SNP interactions. Note that the backbone ${ }^{15} \mathrm{~N}-{ }^{1} \mathrm{H}$ cross-peaks all have very narrow chemical shift dispersion along ${ }^{1} \mathrm{H}$ dimension $(<1 \mathrm{ppm})$, which is characteristic for intrinsically disordered proteins because of their dynamic nature. 

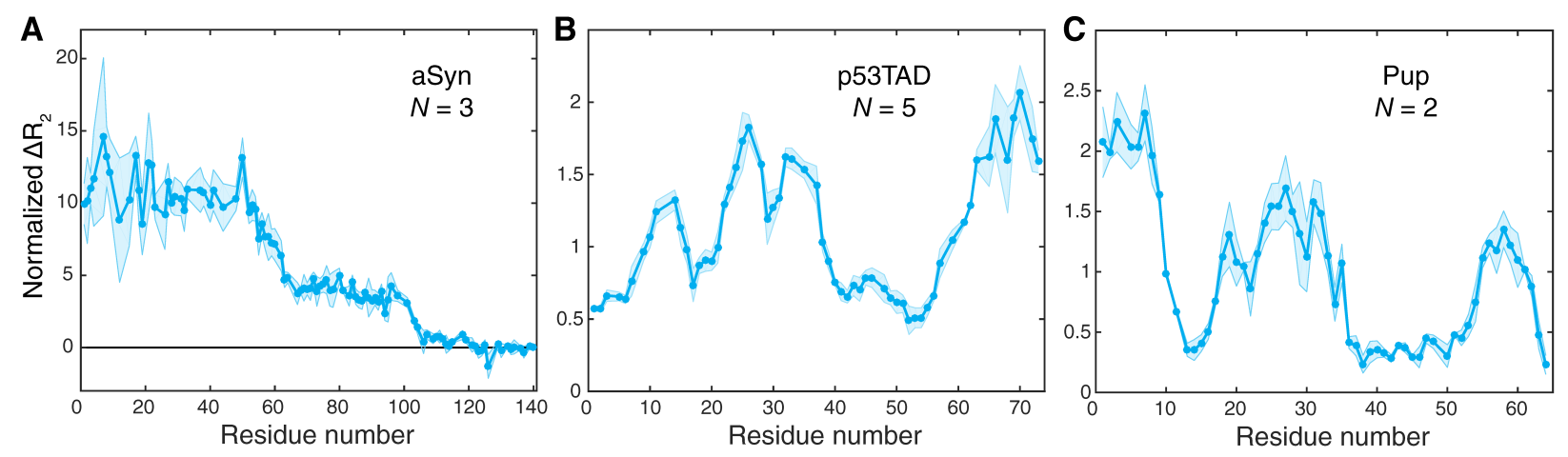

Figure S2. Experimental reproducibility for wild-type (A) aSyn, (B) p53TAD, and (C) Pup based on the $\Delta R_{2}$ profiles of $N$ sets of independently prepared samples. Data were normalized to the standard SNP concentration of $0.17 \mu \mathrm{M}$. Solid circles are the mean $\Delta R_{2}$ values of $N$ data points at each residue site and the boundaries of the shaded area demarcate $1 \times \sigma$ (one standard deviation). Good reproducibility is generally obtained, especially for the regions with lower $\Delta R_{2}$ values because of only small line broadening of the associated ${ }^{15} \mathrm{~N}-{ }^{1} \mathrm{H}$ cross-peaks in the presence of SNPs, leading to a high signal-to-noise ratio. Large $\sigma$ typically results from either inherently low signal-to-noise (especially at higher SNP concentrations) or partially overlapped cross-peaks. 


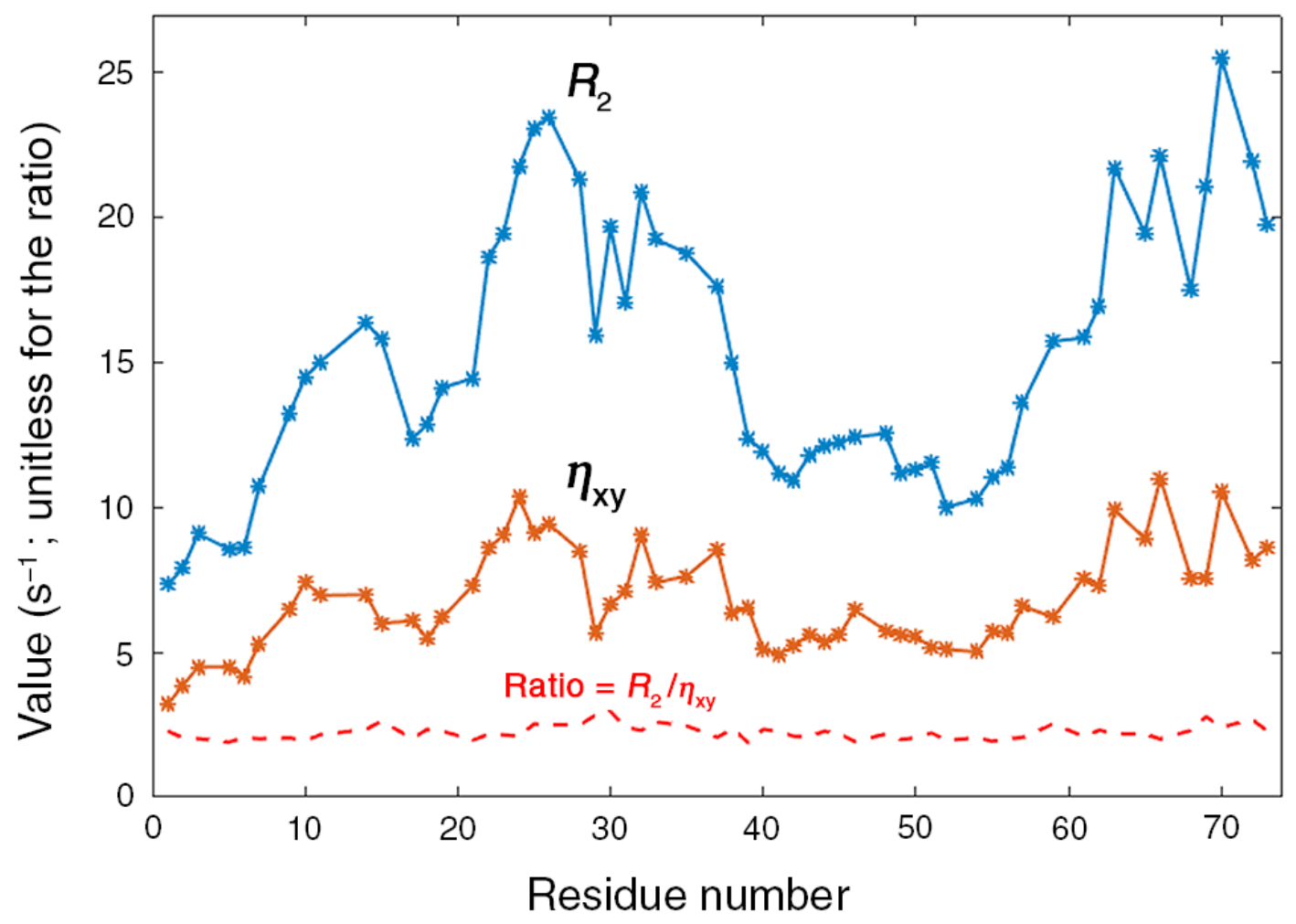

Figure S3. Experimental residue-specific backbone ${ }^{15} \mathrm{~N}-R_{2}$ relaxation (blue) and dipole-CSA crosscorrelated ${ }^{15} \mathrm{~N}-{ }^{1} \mathrm{H} \eta_{\mathrm{xy}}$ relaxation (orange) profiles of p53TAD. The NMR sample consisted of $170 \mu \mathrm{M}$ p53TAD and $1.5 \mu \mathrm{M}$ SNPs. The overall scaling factor $<R_{2} / \eta_{\mathrm{xy}}>=2.235 \pm 0.247$ (red dashed line, bottom) is expected to be larger than 1, since according to Bloch-Wangsness-Redfield theory the expressions for $R_{2}$ and $\eta_{\mathrm{xy}}$ have different prefactors and different spectral density terms. Besides the different overall scaling, the two profiles display very similar segmental variations along the primary sequence consistent with $k_{\mathrm{ex}} \gg \Delta \mathrm{R}_{2}$. It is noted that residue-by-residue variations in $R_{2} / \eta_{\mathrm{xy}}$ are expected, since the chemical shielding anisotropy $\Delta \sigma$ as well as the angle between the CSA principal axis and the $\mathrm{N}-\mathrm{H}$ bond vector can vary between residues. 

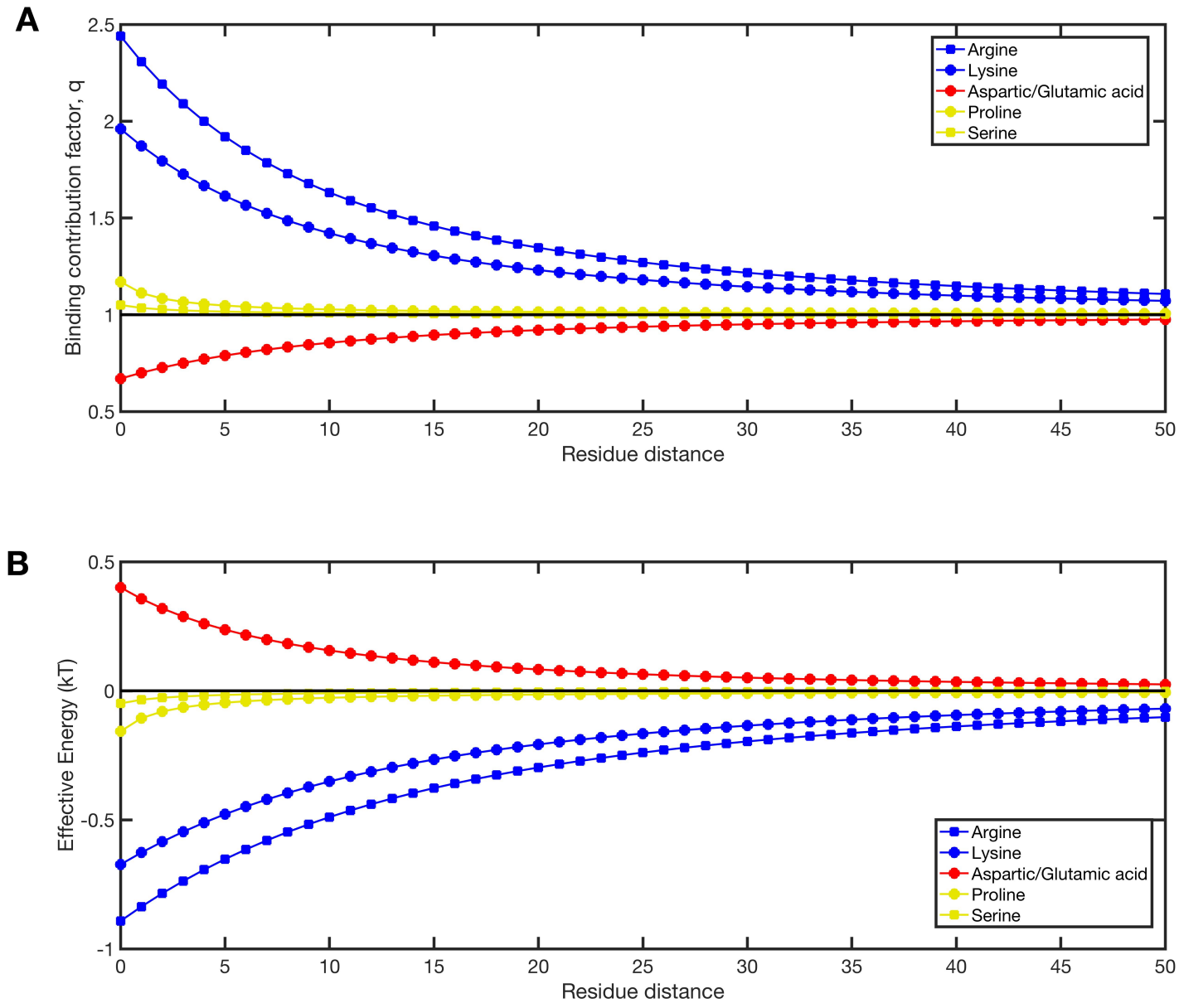

Figure S4. Binding contribution factors (BCF) $q_{n, n-i \mid}$ of Eq. (10) and Figure 2 (Panel S4A) plotted as cooperativity free energies $\Delta G_{n, n-i \mid}=-k_{B} T \ln q_{n, n-i \mid}\left(\right.$ in units of $\left.\mathrm{k}_{\mathrm{B}} \mathrm{T}\right)($ Panel S4B). 


\section{References}

[1] M. Xie, A.L. Hansen, J. Yuan, and R. Brüschweiler. Residue-specific interactions of an intrinsically disordered protein with silica nanoparticles and their quantitative prediction. J. Phys. Chem. C 2016, 120, 24463.

[2] M. Xie, D.-W. Li, J. Yuan, A.L. Hansen, and R. Brüschweiler. Quantitative binding behavior of intrinsically disordered proteins to nanoparticle surfaces at individual residue level. Chem. Eur. J. 2018, 24, 16997.

[3] B. Zhang, M. Xie, L. Bruschweiler-Li, K. Bingol, and R. Brüschweiler. Use of charged nanoparticles in NMR-based metabolomics for spectral simplification and improved metabolite identification. Anal. Chem. 2015, 87, 7211. 